\title{
ASCA SKY SURVEY OBSERVATIONS AND THE COSMIC X-RAY BACKGROUND IN 2-10 KEV
}

\author{
H. INOUE, T. TAKAHASHI, Y. UEDA AND A. YAMASHITA \\ Institute of Space and Astronautical Science, Kanagawa 229 \\ Y. ISHISAKI \\ Tokyo Metropolitan University, Tokyo 192-03 \\ AND \\ Y. OGASAKA \\ NASA Godard Space Flight Center, MD 20771
}

\section{Introduction}

The X-ray background in the energy range above $2 \mathrm{keV}$ is highly uniform except for an excess component along the Galactic plane. The excess along the plane is considered to be associated with our Galaxy, whereas the rest of the emission is believed to be of extragalactic origin. In this paper, the $\mathrm{X}$-ray background at high Galactic latitude is discussed and is designated as the CXB (cosmic X-ray background) to distinguish it from the Galactic origin.

The most likely explanation of the CXB is that the observed CXB flux is supplied by a collection of unresolved, weak discrete sources. The deepest sky survey of X-ray sources in $0.5-2 \mathrm{keV}$ was done by ROSAT (Hasinger et al. $1993 ; 1994)$. The contribution to the $1-2 \mathrm{keV} \mathrm{CXB}$ from sources with flux above $2.5 \times 10^{-15} \mathrm{erg} \mathrm{cm}^{-2} \mathrm{~s}^{-1}$ in $0.5-2 \mathrm{keV}$ is estimated to be about $60 \%$. The discrete source contribution to the CXB in $2-10 \mathrm{keV}$ were so far estimated through the deep survey observations with HEAO-1 A2 (Piccinotti et al. 1982) and with Ginga (Kondo 1992; Hayashida 1990). These results show that the contribution to the CXB in $2-10 \mathrm{keV}$ from sources with flux above $10^{-12} \mathrm{erg} \mathrm{cm}^{-2} \mathrm{~s}^{-1}$ is $\sim 10 \%$ and that the averaged spectral slope is still significantly steeper than that of the CXB.

ASCA carries nested thin-foil X-ray mirrors with an spatial resolution of about an arcminute even in the $2-10 \mathrm{keV}$ band. This enables us to 
observe very faint sources with flux down to several times $10^{-14} \mathrm{erg} \mathrm{cm}^{-2}$ $\mathrm{s}^{-1}$ in $2-10 \mathrm{keV}$ for the first time and to resolve a larger fraction of the CXB into a number of faint sources than previously possible.

\section{ASCA sky survey}

Two kinds of sky survey observations have been being done with ASCA. Large sky survey (LSS): 76 pointing observations with mean exposure of about $30 \mathrm{ks}$ were performed to cover a fairly wide field of sky ( $\sim 5.4 \mathrm{Sq}$. Deg.) near the north Galactic pole.

Deep sky survey (DSS): ASCA was pointed to some sky fields (the SA 57 field, the Lockman Hole field, the Lynx field and so on) for a hundred to several hundreds ks.

In parallel to the LSS and DSS observations, a serendipitous source survey is being done for the ASCA public archive data and it is called as the ASCA medium sensitivity sky survey (MSS). Form the public archive data before Aug. 1995, 481 pointed fields satisfying some criteria ( $|\mathrm{b}|>10^{\circ}$, net exposure $>10 \mathrm{ks}$, the primary target $<10 \mathrm{c} / \mathrm{s} / \mathrm{GIS}$ ) were selected and 992 sources were serendipitously detected in the GIS fields. The catalogue of these sources will soon be open to public as the GIS Source Catalogue (Ver.1) via WWW (Ishisaki et al. 1997).

\section{3. $\log \mathbf{N}-\log \mathrm{S}$ relation in $2-10 \mathrm{keV}$}

Figure 1 shows the $\log \mathrm{N}-\log \mathrm{S}$ relations in $2-10 \mathrm{keV}$ obtained from the DSS mainly in the SA57 field (Ogasaka et al. 1997), and from the LSS (Ueda et al. 1997). The results are consistent with an extrapolation of a $\mathrm{N} \propto$ $\mathrm{S}^{-3 / 2}$ relation from the results of the Ginga fluctuation analysis (Hayashida 1990), the Ginga high-latitude survey (Kondo 1992) and the HEAO-1 A2 survey (Piccinotti et al. 1982). If we integrate source-fluxes down to the ASCA DSS limit of $\sim 4 \times 10^{-14} \mathrm{erg} \mathrm{cm}^{-2} \mathrm{~s}^{-1}$, it is $40 \%$ of the CXB at most.

The $2-10 \mathrm{keV} \log \mathrm{N}-\log \mathrm{S}$ relation is also obtained from the MSS and the result is consistent with those from the LSS and DSS (Ishisaki et al. 1997). Furthermore, the analysis of the angular fluctuation of the CXB is in progress. Although the result is still preliminary, we can say that the nonfluctuating component in the CXB is $20 \%$ at most and that the discrete source contribution to the CXB should be more than $80 \%$.

Fig.2 shows the average of the spectral photon indices of sources in 2 $10 \mathrm{keV}$ in the flux range of $(1-4) \times 10^{-13} \mathrm{erg} \mathrm{cm}^{-2} \mathrm{~s}^{-1}$ detected in the LSS as a function of the source flux, in comparison with that obtained in the higher flux range by the fluctuation analysis of the Ginga background (Hayashida 1990). The average spectral index of sources detected in the 


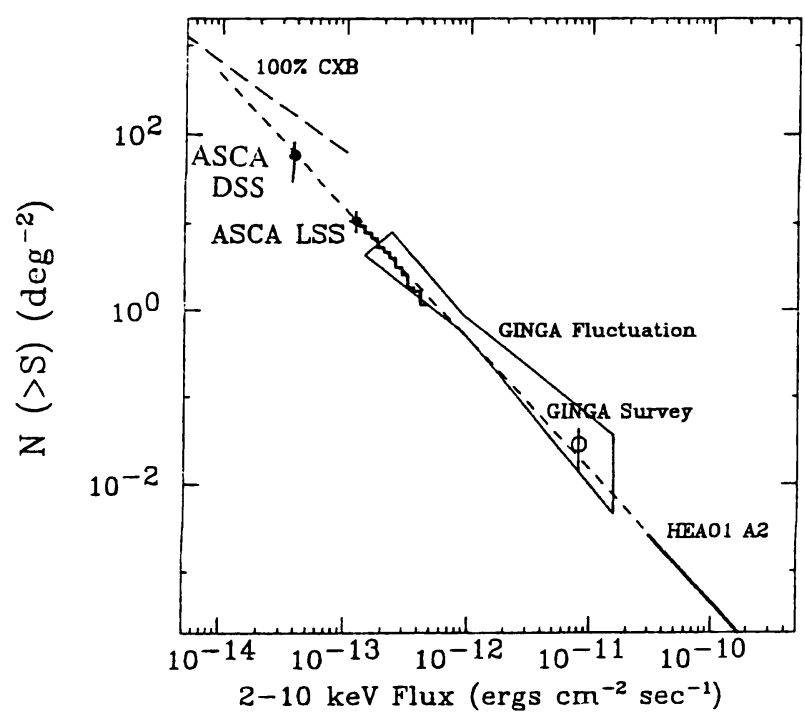

Figure 1. The $\log \mathrm{N}-\log \mathrm{S}$ relation of $\mathrm{X}$-ray sources in $2-10 \mathrm{keV}$ obtained from the DSS observation (Ogasaka et al. 1997), and from the LSS observation (Ueda et al. 1997). The dimmer and brighter points are from the DSS and from the LSS, respectively. A dash-dotted line is an extrapolation of a $\mathrm{N} \propto \mathrm{S}^{-3 / 2}$ relation from the results of Ginga fluctuation analysis (Hayashida 1990), Ginga high-latitude survey (Kondo 1992) and HEAO-1 A2 survey (Piccinotti et al. 1982).

ASCA LSS is significantly harder and closer to the CXB index than that obtained from the previous measurements in the much higher flux range in this energy band. This result implies that a population of sources with an average index similar to the CXB begins to dominate in the flux range around $10^{-13} \mathrm{erg} \mathrm{cm}^{-2} \mathrm{~s}^{-1}$ in $2-10 \mathrm{keV}$.

\section{What are the dim, hard sources?}

Some DSS observations were done in fields overlapped with fields in which deep ROSAT observations and the optical follow-up observations were done (Lockman Hole: Hasinger et al. 1997; Schmidt et al. 1997. GSGP4, QSF3, F855: Georgantopoulos et al. 1997; Boyle et al. 1997).

In the Lockman Hole observations, sources detected with ASCA in 2-10 $\mathrm{keV}$ in the field overlapped with the ROSAT field are almost all identified with ROSAT sources to which optical counterparts were found. Almost all the optical counterparts $(11 / 12)$ are classified as AGNs by Schmidt et al. (1997) and their luminosities and the cosmological redshifts distribute

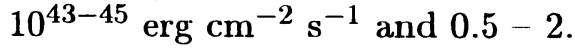

The ratios of the $0.5-2 \mathrm{keV}$ ROSAT flux to the $2-10 \mathrm{keV}$ ASCA flux largely scatter by an order of magnitude and several sources have the 


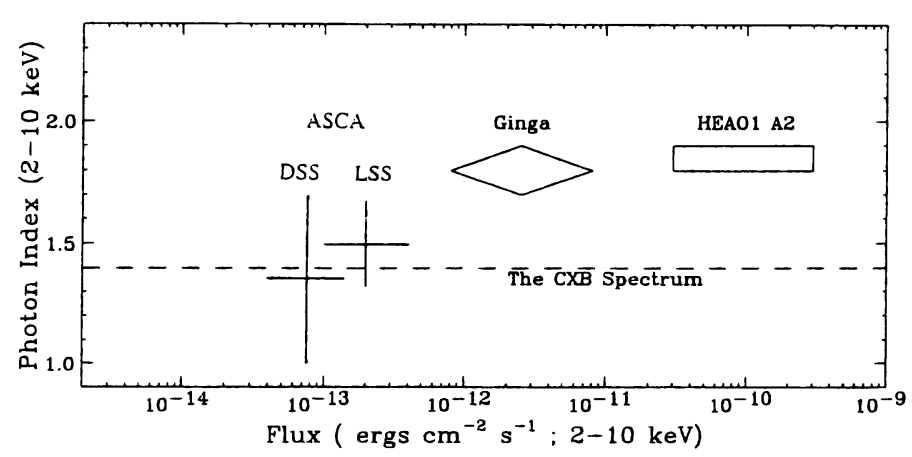

Figure 2. The average spectral photon index of sources detected in the lowest flux range of the LSS (Ueda et al. 1997) and of the DSS (Ogasaka et al. 1997), in comparison with those obtained in the higher flux range: the results of the fluctuation analysis of the Ginga background (Hayashida 1990) and of the Piccinotti sample by HEAO-1 A2. The spectral index of the cosmic X-ray background is also indicated.

ratio as low as 0.1 . The low $0.5-2 \mathrm{keV}$ flux relative to the $2-10 \mathrm{keV}$ flux suggests that those AGNs would suffer from heavy obscuration. In fact, some of them show no optical broad line (Schmidt et al. 1997).

Other optical follow-up observations of the ASCA DSS or LSS sources are also in progress. An observation of a ASCA source in the SA57 field discovered a type- 2 quasar at $\mathrm{z} \simeq 0.9$ (Ohta et. al. 1996). Another optical observation also found a type-2 Seyfert galaxy at the position of a very hard source in the LSS field (Sakano et al. 1997; Akiyama et al. 1997). These all seem to support the idea that most of the dim, hard sources would be highly obscured AGNs at the cosmological distance.

The authors would like to thank G. Hasinger for his providing us the results of the ROSAT Lockman Hole observation.

\section{References}

Akiyama, M., et al., 1997, this Symposium.

Boyle, B.J., et al., 1997, preprint.

Georgantopoulos, I., et al., 1997, preprint.

Hasinger, H., et al., 1993, A\&Ap 275, 1.

Hasinger, H., et al., 1994, A\&Ap 291, 348(Erratum).

Hasinger, H., et al., 1997, preprint.

Hayashida, K., 1990, Ph.D.Thesis, Univ. of Tokyo.

Ishisaki, Y., et al., 1997, this Symposium.

Kondo. H, 1992, Ph.D.Thesis, Univ. of Tokyo.

Ogasaka, Y., et al. 1997, in preparation.

Ohta, K., 1996, Ap.J., 458, L57.

Sakano, M., et al., 1997, in preparation.

Schmidt, M., et al., 1997, preprint.

Ueda, Y., et al. 1997, preprint. 